\title{
At the Crossroad with Morbidity and Mortality Conferences: Lessons Learned through a Narrative Systematic Review
}

\author{
Xin Xiong, ${ }^{1}$ Teela Johnson, ${ }^{2}$ Dev Jayaraman, ${ }^{3,4}$ Emily G. McDonald, ${ }^{5}$ \\ Myriam Martel, ${ }^{6}$ and Alan N. Barkun ${ }^{6,7}$ \\ ${ }^{1}$ Department of Gastroenterology, University of Toronto, Toronto, ON, Canada M5G 2C4 \\ ${ }^{2}$ Department of Family and Community Medicine, University of Toronto, Toronto, ON, Canada M5G 1V7 \\ ${ }^{3}$ Department of Internal Medicine and Department of Critical Care, McGill University Health Center, \\ Montreal, QC, Canada H3G 1A4 \\ ${ }^{4}$ Jewish General Hospital, Montreal, QC, Canada H3T 1E2 \\ ${ }^{5}$ Department of Internal Medicine, McGill University Health Center, Montreal, QC, Canada H3G 1A4 \\ ${ }^{6}$ Division of Gastroenterology, McGill University Health Center, McGill University, Montreal, QC, Canada H3G 1A4 \\ ${ }^{7}$ Epidemiology and Biostatistics and Occupational Health, McGill University Health Center, \\ McGill University, Montreal, QC, Canada H3G 1A4
}

Correspondence should be addressed to Alan N. Barkun; alan.barkun@muhc.mcgill.ca

Received 8 September 2015; Accepted 19 September 2015

Copyright @ 2016 Xin Xiong et al. This is an open access article distributed under the Creative Commons Attribution License, which permits unrestricted use, distribution, and reproduction in any medium, provided the original work is properly cited.

Objective. To determine the process and structure of Morbidity and Mortality Conference (MMC) and to provide guidelines for conducting MMC. Methods. Using a narrative systematic review methodology, literature search was performed from January 1, 1950, to October 2, 2012. Original articles in adult population were included. MMC process and structure, as well as baseline study demographics, main results, and conclusions, were collected. Results. 38 articles were included. 10/38 (26\%) pertained to medical subspecialties and 25/38 (66\%) to surgical subspecialties. 15/38 (40\%) were prospective, 14/38 (37\%) retrospective, 7/38 (18\%) interventional, and 2/38 (5\%) cross-sectional. The goals were quality improvement and education. Of the 10 medical articles, MMC were conducted monthly $60 \%$ of the time. Cases discussed included complications (60\%), deaths (30\%), educational values (30\%), and system issues (40\%). Recommendations for improvements were made frequently (90\%). Of the 25 articles in surgery, MMCs were weekly (60\% of the time). Cases covered mainly complications (72\%) and death (52\%), with fewer cases dedicated to education (12\%). System issues and recommendations were less commonly reported. Conclusion. Fundamental differences existed in medical versus surgical departments in conducting MMC, although the goals remained similar. We provide a schematic guideline for MMC through a summary of existing literature.

\section{Introduction}

Morbidity and Mortality Conferences (MMCs) are held ubiquitously throughout medical services worldwide [1-6]. Historically, they became an integral component of surgical departments in the early 1900s, following conferences on hospital standardization $[7,8]$ and introduction of the "End Result System" by Ernst Codman who was first to systematically record and review patient demographics and related adverse events [3,9]. Since the publication of To Err is Human [6], MMCs continue to be a widespread practice in medical training programs and are designed to "identify medical errors in order to learn from them to improve medical practice" [3].

The Accreditation Council for Graduate Medical Education (ACGME) has incorporated mandatory MMC in each training program since 1983 [10, 11]. Furthermore, the majority of hospitals require MMC in order to maintain accreditation. Over time the focus has now shifted towards identifying and correcting system-related issues through the evolving field of quality improvement, as opposed to assigning blame and responsibility to the individual [12-14].

Despite efforts to unify MMC format, their contents remain heterogeneous $[1,8]$, with no clear guidelines for 
execution. For example, there is a dichotomy of practice between medical and surgical departments $[1,5]$, with differing recommendations from the ACGME [10, 11]. Surgical ACGME requires weekly MMC to be performed, whereas a frequency has not been specified in the ACGME for most medical subspecialties. In addition, the case selection process for both is largely unspecified. In some studies, cases are selected from a list of voluntarily reported morbidities $[15$, 16], whereas, in others, they are selected from predefined complication registries [17].

The goal of this paper is twofold. First goal is to determine, through a narrative systematic review of the literature, the process and content of MMC in medical and surgical departments. Second goal is to provide a schematic guideline to improve the organization of these conferences based on the available literature.

\section{Methods}

2.1. Search Strategy. We performed a computerized medical literature search from January 1, 1950, to October 2, 2012, using OVID MEDLINE, EMBASE, CENTRAL, Scopus, and ISI Web of knowledge 5.6. We selected articles using a search strategy with a combination of MeSH headings and text keywords related to (1) mortality or morbidity and (2) medical education, teaching rounds, conferences, or presentation. We carried out recursive search and cross-referencing using a "similar articles" function. We also identified articles through hand searches after the initial search. We included all original studies on adult population focused on the discussion of the MMC, in French or English. Studies with original data regarding multiple aspects of MMC were assessed. We excluded articles with only abstract publication or conference presentations because these do not provide sufficient information for the purpose of this review. We reviewed national surveys, but we did not collect their data for analysis in this systematic review. Duplicates were excluded. Two investigators (Xin Xiong and Teela Johnson/Alan N. Barkun of the authors) assessed all articles according to the selection criteria independently; disagreements were discussed until a consensus was reached.

2.2. Choice of Outcomes and Variables of Interest. In the current literature, there exists a variety of organized terminologies to describe different aspects of MMC. In our study, we adjudicated each article's main focus into one of the following categories: goals, structure, or process [2]. For the purposes of this review, the definition for each of these categories was adapted from the following concepts described by Aboumatar et al. [2] (Figure 1). Goal is the objective achieved by conducting MMC. Structure characterizes how MMC is carried out; this includes MMC frequency, duration, number of cases presented, and participants (moderator, presenters, and audience). Process indicates the case selection, analysis, literature review, and proposal for improvement. Whether recommendations were implemented as a result of MMC discussion was also noted. In addition, we also collected information with respect to each study's setting, discipline, study methodology, stated objectives, and outcomes as well

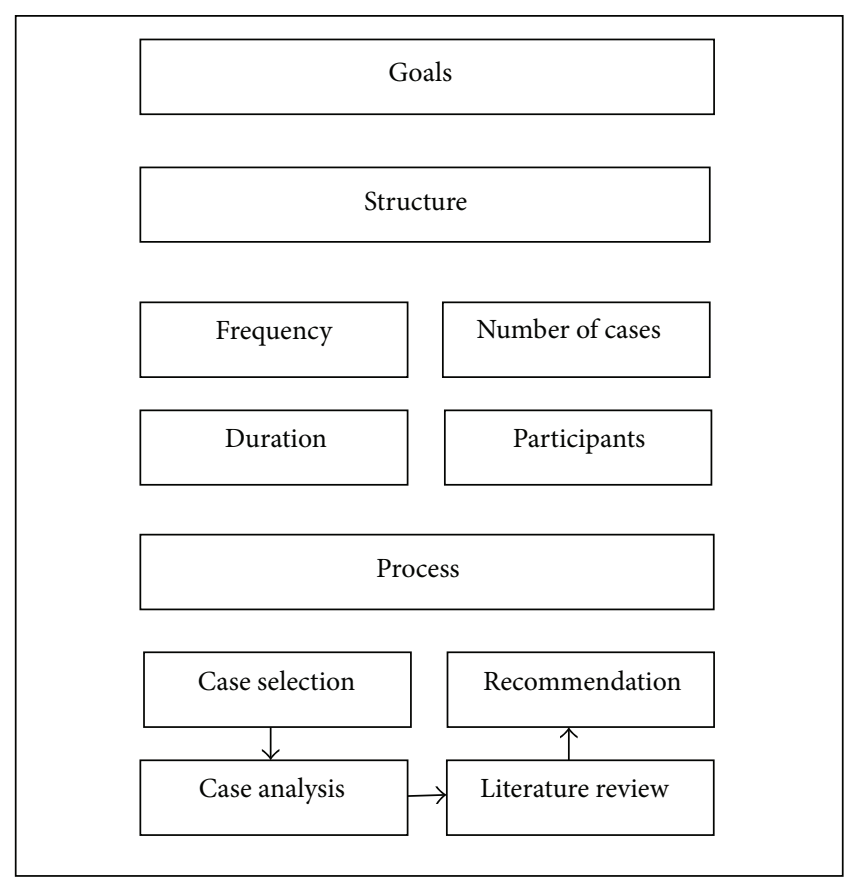

FIGURE 1: Proposed characteristics of MMC, modified with permission from Aboumatar et al. [2].

as how these were measured. Given that the ACGME has specified different requirements for medical and surgical specialties regarding MMC $[10,11]$, we collected and analyzed these data separately.

2.3. Sources of Possible Heterogeneity. Comparative qualitative analyses were performed across studies to assess the clinical homogeneity of study populations (cases, patients, or health care professionals), interventions, and outcomes. Statistical heterogeneity was not evaluated as most outcomes were qualitative in nature.

\section{Results}

3.1. Included Studies. From a total of 405 citations identified, 358 were excluded because they did not pertain to discussion of aspects of MMC, 8 were excluded given they were either national surveys or review articles, 3 were excluded because they did not address adult populations, and 3 were excluded due to insufficient information. Cross-referencing yielded 5 additional articles. Therefore, 38 studies were included (see Figure 2).

3.2. Synthesis of Literature. Tables 1(a), 1(b), and 1(c) provide a summary of the 38 studies included in this narrative systematic review. Ten articles pertain to departments or divisions of medicine (including internal medicine and its subspecialties, primary care, and critical care), 25 to surgery (which includes surgery and its subspecialties, obstetrics, and anesthesia), and 3 to both medicine and surgery. These tables highlight the heterogeneity amongst studies existing in 


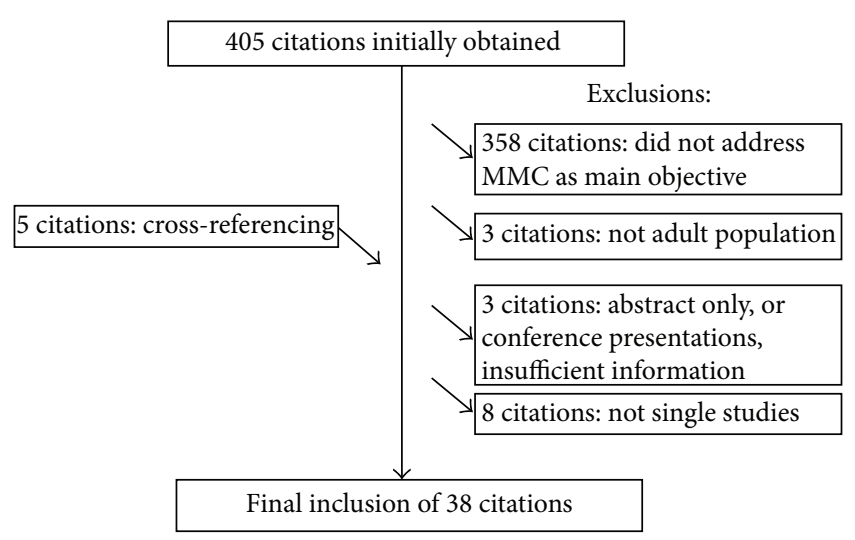

FIGURE 2: QUOROM diagram.

the literature. The majority of studies were performed in academic centers (34/38 or $89 \%)$ ): $15 / 38(40 \%)$ were prospective studies and $14 / 38$ (37\%) retrospective; $2 / 38(5 \%)$ used a crosssectional design, while $7 / 38(18 \%)$ were interventional. Of note, articles addressing surgical departments tended to be more quantitative than those studying medical departments. In addition, there were no uniform definitions of the various aspects of MMC (goals, structure, and process) and there was no homogenous method for measurement of errors across studies.

Overall, the focus (goal, structure, and process) that these 38 articles have covered (numbers not mutually exclusive) is as follows: $30 / 38$ articles (79\%) discussed the goal of the MMC, $30 / 38$ (79\%) the structure, and $26 / 38(68 \%)$ the process. 10/38 articles (26\%) discussed goal and structure, $2 / 38(5 \%)$ goal and process, and $6 / 38(16 \%)$ structure and process. 14/38 articles (37\%) encompassed all 3 categories.

3.3. Medicine. Figure 3 demonstrates the details of characteristics of MMC in medicine. In summary, from the review of 10 articles, the goal appeared to be quality improvement in $90 \%$ and education in $40 \%$ (percentages are not mutually exclusive). The frequency was most often monthly (60\%). The duration most often spanned 1 hour (50\%). Participants included faculty, residents, nurses, other health care professionals, and staff of different specialties. Usually, cases were presented by residents $(40 \%)$ and less often by faculty (30\%). In $70 \%$ of cases, the moderator was a faculty member. The cases were all selected before MMC, most often by faculty (40\%). Cases frequently addressed complications $(60 \%)$. Only $20 \%$ of articles reported a requirement of a literature review, but $90 \%$ reported implementation of recommendation. A more detailed tabular description of the rounds' content is shown in Figure 3, adopting the proposed MMC study characteristics identified in Figure 1.

3.4. Surgery. Figure 4 presented the details of characteristics of MMC in surgery. In summary, after reviewing 25 articles, the goals seemed to be predominantly targeting education $(60 \%)$ or quality improvement $(56 \%)$. The frequency was most often weekly (60\%). The duration of the MMC was most often not reported (60\%), but, when documented, most
MMC lasted 1 hour (28\%). Participants included faculty, residents, nurses, other health care professionals, and staff of different specialties. Usually, cases were presented by residents $(60 \%)$. In $52 \%$ of cases, the moderator was a faculty. The cases were all selected before MMC, most often by faculty $(20 \%)$ or dedicated team members $(20 \%)$. Cases were selected if they addressed complications (70\%), including death in $52 \%$. Only $40 \%$ of articles reported the requirement of a literature review to support the MMC (see Figure 4).

As can be noted from Figures 3 and 4, there are differences between MMC performed in medical and surgical departments. In medical departments, MMCs are more often monthly whereas in surgery they are weekly. Medical MMCs present fewer cases, with a greater focus on discussion and analysis of systems issues, with the goal of providing recommendations for improvements.

\section{Discussion}

Upon review of the available literature on MMC, it is apparent that there is considerable heterogeneity in the content and goals of MMC across both medical and surgical services. This heterogeneity has been shown to limit the effectiveness of MMC [3, 15, 32, 42, 51]. Through the current review, we observe an important lack of standardization and precision of the definitions and terms used to describe different aspects of MMC (including goal, process, and structure). In many studies, what we believe to be important characteristics of MMC have not been recorded consistently, giving rise to only a few quality articles, leading us to believe that the reported outcomes are less generalizable. Because of this lack of rigorous reporting and poorly generalizable data, a synthesis of the literature is challenging. However, we are still able to infer several helpful conclusions regarding the process and content of MMC.

To begin with, we note that medical and surgical departments have different approaches to the process of MMC, which has been confirmed by previous reviews $[1,5,51]$ and national surveys $[3,51,52]$. In medicine and its subspecialties, the goal of these conferences appears more focused towards quality improvement, whereas, in surgery, education and quality improvement are more balanced. Surgical departments comply with the ACGME MMC frequency requirement [11], with weekly meetings. In comparison, possibly because no such frequency guideline exists for [10] medical departments, MMCs are often done on a monthly basis. Moreover, surgical departments present more cases per MMC. Combining this with increased frequency, more cases are presented in surgery compared to medicine $[1,4,5]$. In contrast, discussion of fewer cases in medicine departments may allow for increased opportunities for discussion of system issues, recommendations, and follow-up of identified problems $[1,2]$.

There exists no direct evidence for a need for differing practices between surgical and medical departments. For example, no empiric data favors presenting more versus less cases; certain studies $[17,41,42,44]$ propose presenting all perioperative complications and mortalities, while other 
TABle 1: (a) Articles for medicine and subspecialties, primary care, and ICU (10 articles). (b) Articles for surgery and its subspecialties, obstetrics, and anesthesia ( 25 articles). (c) Articles for both medicine and surgery ( 3 articles).

(a)

\begin{tabular}{lccc}
\hline Study/setting & Stated objective & Category & Type of study \\
\hline $\begin{array}{l}\text { Kirschenbaum et al., } \\
2010[18]\end{array}$ & $\begin{array}{c}\text { Determining if audit of } \\
\text { patients plus a focused } \\
\text { MMC }\end{array}$ & Goal & Interventional: before and \\
Academic & care in ICU & after survey
\end{tabular}

\begin{tabular}{|c|c|c|c|}
\hline $\begin{array}{l}\text { Ksouri et al., } 2010 \text { [19] } \\
\text { ICU } \\
\text { Academic }\end{array}$ & $\begin{array}{l}\text { Evaluating MMC in ICU } \\
\text { for improving quality of } \\
\text { care and patient safety }\end{array}$ & $\begin{array}{l}\text { Goal, } \\
\text { structure, } \\
\text { process }\end{array}$ & Retrospective \\
\hline
\end{tabular}

\begin{tabular}{|c|c|c|c|}
\hline $\begin{array}{l}\text { Kuper et al., } 2010 \text { [20] } \\
\text { Academic }\end{array}$ & $\begin{array}{l}\text { Exploring the role of } \\
\text { MMC in medical } \\
\text { education }\end{array}$ & $\begin{array}{l}\text { Goal, } \\
\text { structure }\end{array}$ & $\begin{array}{c}\text { Prospective/ethnographic } \\
\text { interviews, evaluation of } \\
\text { notes, and audiotape of } \\
\text { MMC }\end{array}$ \\
\hline
\end{tabular}

Main results and conclusion
MMCs result in improved rapid response
and hospital outcomes (number of
cardiac arrests decreased from 3.1/1000 to
$0.6 / 1000, p=0.002$, deaths decreased
from $34 / 1000$ to $24 / 1000, p=0.024$ ).
from $34 / 1000$ to $24 / 1000, p=0.024)$.

MMCs provide educational value and can be used to assess quality of care, patient safety, and interpersonal and team communication.

MMCs are effective vehicles to address competencies in patient safety and quality improvement. A disjunction between teaching valued by staffs and learning valued by students were noted.

MMCs with system audit have higher

Szostek et al., 2010

[21]

$\begin{array}{ccc}\begin{array}{c}\text { Determining } \\ \text { educational value of } \\ \text { system audit }\end{array} & \begin{array}{c}\text { Goal, } \\ \text { structure, } \\ \text { process }\end{array} & \begin{array}{c}\text { Interventional: before and } \\ \text { after survey }\end{array}\end{array}$

educational values, $95 \%$ (versus $61 \%$ preimplementation) and stimulating increased interest in education as well as ensuring improved quality of care.

New MMC format allows good educational forum with increased Bechtold et al., 2008 [22]

Describing new MMC experience
Goal, Interventional: before and structure, process after survey
Proposing a format as a model for MMC in academic center for gastroenterology 2008 [23]

Academic

\section{Structure} process

(Goal,

Goldszer et al., 2006

[24]

Community

Describing MMC in
primary care center

Evaluating the role in

Kravet et al., 2006

[25]

Academic
Goal,

structure, process participation. Educational intervention and recommendations were more likely to be carried out.

Overall complication rate of $0.76 \%$, within that reported in the literature. Monthly MMCs are a means of monitoring patient care and enhancing trainee education.

The MMC format is a useful tool to improve patient care.

\begin{tabular}{|c|c|c|c|c|}
\hline \multicolumn{5}{|c|}{ Round } \\
\hline $\begin{array}{l}\text { Denis et al., } 2003 \text { [17] } \\
\text { Community }\end{array}$ & $\begin{array}{l}\text { MMC format assessed as } \\
\text { a quality improvement } \\
\text { tool in gastroenterology }\end{array}$ & Goal, process & Prospective: chart review & $\begin{array}{l}\text { Systematic prospective recording of } \\
\text { complications and careful exhaustive } \\
\text { retrospective analysis during MMC are } \\
\text { efficient and complementary tools for } \\
\text { continuous quality improvement. }\end{array}$ \\
\hline $\begin{array}{l}\text { Esselman and } \\
\text { Dillman-Long, } 2002 \\
\text { [26] } \\
\text { Academic }\end{array}$ & $\begin{array}{l}\text { Refocusing MMC onto } \\
\text { system issues and } \\
\text { avoiding placing blame } \\
\text { on individuals }\end{array}$ & $\begin{array}{l}\text { Goal, } \\
\text { structure, } \\
\text { process }\end{array}$ & Retrospective & $\begin{array}{l}\text { MMCs are important in quality } \\
\text { improvement when focusing on system } \\
\text { issues. }\end{array}$ \\
\hline
\end{tabular}

MMCs in Grand Rounds are effective Goal,
structure

(b)

\begin{tabular}{|c|c|c|c|c|c|}
\hline Study & $\begin{array}{c}\text { Setting } \\
\text { discipline }\end{array}$ & Stated objective & Category & Type of study & Main results and conclusion \\
\hline $\begin{array}{l}\text { Falcone and } \\
\text { Watson, } 2012 \\
{[27]}\end{array}$ & $\begin{array}{l}\text { Academic } \\
\text { surgery }\end{array}$ & $\begin{array}{l}\text { Assessing participation } \\
\text { and cost benefit of } \\
\text { teleconference in MMC }\end{array}$ & Goal, structure & $\begin{array}{c}\text { Retrospective } \\
\text { cost-effective analysis }\end{array}$ & $\begin{array}{l}\text { Teleconferencing allows for } \\
\text { increased faculty attendance at } \\
\text { MMC ( } 5 \text { per conference, } \\
p<0.001 \text { ) and is cost-effective } \\
\text { (annual net savings of } 7624 \$ \text { ). }\end{array}$ \\
\hline
\end{tabular}


(b) Continued.

\begin{tabular}{|c|c|c|c|c|c|}
\hline Study & $\begin{array}{c}\text { Setting } \\
\text { discipline }\end{array}$ & Stated objective & Category & Type of study & Main results and conclusion \\
\hline $\begin{array}{l}\text { Falcone et al., } \\
2012[28]\end{array}$ & $\begin{array}{l}\text { Academic } \\
\text { surgery }\end{array}$ & $\begin{array}{l}\text { Describing reporting } \\
\text { patterns of general } \\
\text { surgery residents. } \\
\text { Describing adverse } \\
\text { events rates compared to } \\
\text { published data }\end{array}$ & Process & Retrospective cohort & $\begin{array}{l}\text { Underreporting of nonfatal } \\
\text { adverse events: } 2.5 \% \text { versus } 4.3 \% \\
\text { reported in literature; majority of } \\
\text { adverse events were from death } \\
(24.1 \%) \text {, hematologic or vascular } \\
\text { complications ( } 16.7 \%) \text {, and } \\
\text { gastrointestinal complications } \\
(16.1 \%) \text {. }\end{array}$ \\
\hline $\begin{array}{l}\text { Thomas et al., } \\
2012[29]\end{array}$ & $\begin{array}{l}\text { Academic } \\
\text { surgery }\end{array}$ & $\begin{array}{l}\text { Integrating minor } \\
\text { complication reporting } \\
\text { in MMC for its } \\
\text { educational value }\end{array}$ & $\begin{array}{l}\text { Goal, } \\
\text { structure, } \\
\text { process }\end{array}$ & $\begin{array}{l}\text { Interventional: before and } \\
\text { after survey }\end{array}$ & $\begin{array}{l}\text { Postimplementation of reporting } \\
\text { of minor adverse outcomes in } \\
\text { MMC; } 95 \% \text { of surveyed } \\
\text { population }(p<0.01) \text { stated that } \\
\text { this provides improved quality } \\
\text { assurance }(71 \%, p<0.05) \text {. }\end{array}$ \\
\hline $\begin{array}{l}\text { Bevis et al., } 2011 \\
{[30]}\end{array}$ & $\begin{array}{l}\text { Academic } \\
\text { obstetrics }\end{array}$ & $\begin{array}{l}\text { Characterizing the } \\
\text { MMC as a cost-effective } \\
\text { and efficient approach } \\
\text { for addressing the } \\
\text { ACGME competencies }\end{array}$ & Goal, structure & Retrospective & $\begin{array}{l}\text { MMCs address } 100 \% \\
\text { practice-based learning and } \\
\text { medical knowledge, } 19 \% \\
\text { systems-based practice, } 10 \% \\
\text { communication, and } 6 \% \\
\text { professionalism or ethics. }\end{array}$ \\
\hline $\begin{array}{l}\text { Kauffmann et } \\
\text { al., } 2011[31]\end{array}$ & $\begin{array}{l}\text { Academic } \\
\text { surgery }\end{array}$ & $\begin{array}{l}\text { Multidisciplinary MMC } \\
\text { presents a unique } \\
\text { opportunity to } \\
\text { incorporate all } 6 \\
\text { ACGME competencies } \\
\text { effectively and efficiently }\end{array}$ & $\begin{array}{l}\text { Goal, structure, } \\
\text { process }\end{array}$ & Retrospective & $\begin{array}{l}\text { Multidisciplinary MMCs are } \\
\text { useful in rapidly achieving } \\
\text { quality improvement while } \\
\text { creating opportunities for system } \\
\text { health care delivery initiatives. }\end{array}$ \\
\hline $\begin{array}{l}\text { Kim et al., } 2010 \\
{[32]}\end{array}$ & $\begin{array}{l}\text { Academic } \\
\text { surgery }\end{array}$ & $\begin{array}{l}\text { Examining the content } \\
\text { and process of MMCs } \\
\text { and testing the } \\
\text { hypothesis that a } \\
\text { structured format can } \\
\text { improve teaching and } \\
\text { learning }\end{array}$ & Goal, structure & $\begin{array}{l}\text { Interventional: before and } \\
\text { after survey }\end{array}$ & $\begin{array}{l}\text { A structured MMC format } \\
\text { improves the identification of the } \\
\text { cause for complication ( } 3.11 \text { to } \\
4.56, p<0.05) .67 \% \text { of surveyed } \\
\text { population expressed an overall } \\
\text { improved experience in quality } \\
\text { of care. }\end{array}$ \\
\hline $\begin{array}{l}\text { Steiger et al., } \\
2010[33]\end{array}$ & $\begin{array}{c}\text { Academic } \\
\text { neurosurgery }\end{array}$ & $\begin{array}{l}\text { Describing methods to } \\
\text { identify critical cases, } \\
\text { the system of analysis, } \\
\text { classification of MMC, } \\
\text { and resulted impact }\end{array}$ & Goal, process & Retrospective & $\begin{array}{l}\text { A reliable system is employed by } \\
\text { MMC to identify cases, } \\
\text { providing good instruments for } \\
\text { quality control and problem } \\
\text { oriented teaching. Impact on } \\
\text { quality improvement remains } \\
\text { questionable. }\end{array}$ \\
\hline $\begin{array}{l}\text { Antonacci et al., } \\
2009[34]\end{array}$ & $\begin{array}{l}\text { Academic } \\
\text { and } \\
\text { community } \\
\text { surgery }\end{array}$ & $\begin{array}{c}\text { Describing } \\
\text { comprehensive surgeon } \\
\text { report card system based } \\
\text { on MMC, in a } \\
\text { nonpunitive error } \\
\text { analysis fashion } \\
\end{array}$ & $\begin{array}{l}\text { Goal, structure, } \\
\text { process }\end{array}$ & Prospective & $\begin{array}{l}\text { MMCs result in a } 40 \% \text { reduction } \\
\text { of gross mortality }(p<0.001) \text {. } \\
\text { Quality issues were identified as } \\
3 \text { times greater than required by } \\
\text { New York State regulations. }\end{array}$ \\
\hline $\begin{array}{l}\text { Berenholtz et } \\
\text { al., } 2009 \text { [12] }\end{array}$ & $\begin{array}{l}\text { Academic } \\
\text { surgery }\end{array}$ & $\begin{array}{c}\text { Describing learning } \\
\text { from a defect tool as a } \\
\text { strategy to meet } \\
\text { ACGME requirements } \\
\text { and enhance traditional } \\
\text { MMCs } \\
\end{array}$ & $\begin{array}{l}\text { Goal, structure, } \\
\text { process }\end{array}$ & Prospective & $\begin{array}{l}\text { MMCs present a helpful strategy } \\
\text { to learn from medical incident } \\
\text { and improve patient safety and } \\
\text { quality of care. Adverse events } \\
\text { are usually failures in the system. }\end{array}$ \\
\hline $\begin{array}{l}\text { Bender et al., } \\
2009[35]\end{array}$ & $\begin{array}{l}\text { Academic } \\
\text { surgery }\end{array}$ & $\begin{array}{c}\text { Determining } \\
\text { heterogeneity of } \\
\text { assessment in } \\
\text { peer-reviewed MMC } \\
\text { and evaluating biases }\end{array}$ & Process & Prospective: survey & $\begin{array}{l}\text { Significant disagreement noted } \\
\text { amongst assessors leading } \\
\text { authors to conclude that the } \\
\text { reliability of peer review is } \\
\text { questionable. }\end{array}$ \\
\hline
\end{tabular}


(b) Continued.

\begin{tabular}{|c|c|c|c|c|c|}
\hline Study & $\begin{array}{c}\text { Setting } \\
\text { discipline }\end{array}$ & Stated objective & Category & Type of study & Main results and conclusion \\
\hline $\begin{array}{l}\text { Dissanaike et al., } \\
2009 \text { [36] }\end{array}$ & $\begin{array}{l}\text { Academic } \\
\text { surgery }\end{array}$ & $\begin{array}{l}\text { Comparing the } \\
\text { perceptions of } \\
\text { preventability of } \\
\text { mortalities and severity } \\
\text { of complications of } \\
\text { MMC attendees } \\
\end{array}$ & $\begin{array}{l}\text { Structure, } \\
\text { process }\end{array}$ & Prospective & $\begin{array}{l}\text { Surgical residents assign higher } \\
\text { severity to trauma-related } \\
\text { complications than other groups. } \\
\text { More objective grading tools are } \\
\text { necessary to improve the } \\
\text { adequacy of MMC. }\end{array}$ \\
\hline $\begin{array}{l}\text { Greco et al., } \\
2009 \text { [37] }\end{array}$ & $\begin{array}{l}\text { Academic } \\
\text { surgery }\end{array}$ & $\begin{array}{l}\text { Describing the authors' } \\
\text { experience with } \\
\text { incorporating a clinical } \\
\text { librarian into the process } \\
\text { of MMCs }\end{array}$ & Goal, structure & Prospective & $\begin{array}{l}\text { The clinical librarian program } \\
\text { has improved the quality of } \\
\text { MMC presentations. }\end{array}$ \\
\hline $\begin{array}{l}\text { Folcik et al., } \\
2007 \text { [38] }\end{array}$ & $\begin{array}{l}\text { Academic } \\
\text { surgery }\end{array}$ & $\begin{array}{l}\text { Describing a two-tiered } \\
\text { process MMC with } \\
\text { dedicated subcommittee } \\
\text { for quality improvement } \\
\text { for ACGME } \\
\text { competencies } \\
\end{array}$ & $\begin{array}{l}\text { Goal, structure, } \\
\text { process }\end{array}$ & $\begin{array}{l}\text { Prospective: reviewed } \\
\text { MMC note, survey }\end{array}$ & $\begin{array}{l}\text { MMCs with a dedicated quality } \\
\text { improvement subcommittee } \\
\text { decrease time to implementation } \\
\text { of changes ( } 3-4 \text { months } \\
\text { compared to } 10-12 \text { months). }\end{array}$ \\
\hline $\begin{array}{l}\text { Prince et al., } \\
2007 \text { [39] }\end{array}$ & $\begin{array}{l}\text { Academic } \\
\text { surgery }\end{array}$ & $\begin{array}{l}\text { Analyzing which } \\
\text { features of MMC } \\
\text { associated with greater } \\
\text { educational value and } \\
\text { increasing confidence in } \\
\text { the future }\end{array}$ & Goal, structure & Prospective: survey & $\begin{array}{l}\text { Audience interaction improves } \\
\text { educational value and increased } \\
\text { confidence in managing complex } \\
\text { problems presented in MMC } \\
(p<0.01) \text {. This is achieved by } \\
\text { increased questioning and } \\
\text { explanation, radiology images } \\
\text { read by presenters, and } \\
\text { moderators facilitating } \\
\text { discussion. }\end{array}$ \\
\hline
\end{tabular}

\begin{tabular}{lcccc} 
Goldfarb and & Community & $\begin{array}{c}\text { Sharing a reproducible } \\
\text { process for presenting, } \\
\text { anaker, 2006 [40] } \\
\text { surgery } \\
\text { surgical morbidity and } \\
\text { mortality }\end{array}$ & $\begin{array}{c}\text { Goal, structure, } \\
\text { process }\end{array}$ & $\begin{array}{c}\text { Retrospective: chart } \\
\text { review }\end{array}$ \\
\hline
\end{tabular}

MMCs help in directing changes to resident training, hospital systems, and surgical practice.

\begin{tabular}{|c|c|c|c|c|c|}
\hline $\begin{array}{l}\text { Hutter et al., } \\
2006[41]\end{array}$ & $\begin{array}{l}\text { Academic } \\
\text { surgery }\end{array}$ & $\begin{array}{l}\text { Comparing data as } \\
\text { reported in a traditional } \\
\text { MMC versus National } \\
\text { Surgical Quality } \\
\text { Improvement Program } \\
\text { (NSQIP) }\end{array}$ & Goal & $\begin{array}{l}\text { Retrospective: MMC data } \\
\text { reviewed }\end{array}$ & $\begin{array}{l}\text { MMCs underreport adverse } \\
\text { events when compared to } \\
\text { NSQIP: } 1 / 2 \text { deaths and } 3 / 4 \\
\text { complications were not } \\
\text { presented, especially in patients } \\
\text { with incurable disease, } \\
\text { transferred care, and "medical" } \\
\text { problems. }\end{array}$ \\
\hline $\begin{array}{l}\text { Miller et al., } \\
2006[42]\end{array}$ & $\begin{array}{l}\text { Academic } \\
\text { urology }\end{array}$ & $\begin{array}{c}\text { Comparing } \\
\text { complications reported } \\
\text { at the MMC versus } \\
\text { NSQIP }\end{array}$ & $\begin{array}{l}\text { Goal, } \\
\text { process }\end{array}$ & $\begin{array}{l}\text { Retrospective: chart } \\
\text { review }\end{array}$ & $\begin{array}{l}\text { MMCs have low sensitivity for } \\
\text { detection of complications } \\
(25 \%) \text {. NSQIP may be better for } \\
\text { urologic quality improvement } \\
\text { endeavors. }\end{array}$ \\
\hline $\begin{array}{l}\text { Rosenfeld et al., } \\
2005[43]\end{array}$ & $\begin{array}{l}\text { Community } \\
\text { surgery }\end{array}$ & $\begin{array}{c}\text { Evaluating new MMC } \\
\text { for ACGME } \\
\text { competencies }\end{array}$ & $\begin{array}{l}\text { Goal, structure, } \\
\text { process }\end{array}$ & $\begin{array}{l}\text { Retrospective: chart } \\
\text { review }\end{array}$ & $\begin{array}{l}\text { The restructuring of MMC so } \\
\text { that a case is analyzed according } \\
\text { to ACGME general competencies } \\
\text { improved general interest and } \\
\text { educational value. MMCs } \\
\text { provide opportunities to teach } \\
\text { ACGME general competencies. }\end{array}$ \\
\hline $\begin{array}{l}\text { Murayama et al., } \\
2002[44]\end{array}$ & $\begin{array}{l}\text { Academic } \\
\text { surgery }\end{array}$ & $\begin{array}{l}\text { Evaluating impact of } \\
\text { changes made to our } \\
\text { MMC (5-10 min case } \\
\text { summary, literature } \\
\text { review, and faculty } \\
\text { discussion with } \\
\text { moderator) }\end{array}$ & Goal, structure & $\begin{array}{l}\text { Interventional: before and } \\
\text { after survey }\end{array}$ & $\begin{array}{l}\text { Surgical residents perceive } \\
\text { significant improvements after } \\
\text { changes to the MMC process. } \\
\text { This is not the case for surgical } \\
\text { staff. }\end{array}$ \\
\hline
\end{tabular}


(b) Continued.

\begin{tabular}{|c|c|c|c|c|c|}
\hline Study & $\begin{array}{c}\text { Setting } \\
\text { discipline }\end{array}$ & Stated objective & Category & Type of study & Main results and conclusion \\
\hline $\begin{array}{l}\text { Risucci et al., } \\
2003[45]\end{array}$ & $\begin{array}{l}\text { Academic } \\
\text { surgery }\end{array}$ & $\begin{array}{l}\text { Assessing interrater } \\
\text { agreement before and } \\
\text { after initiation of a } \\
\text { modified MMC } \\
\text { (presentation of } 3 \text { cases } \\
\text { of } 30 \text { minutes with } \\
\text { literature review) }\end{array}$ & $\begin{array}{l}\text { Structure, } \\
\text { process }\end{array}$ & $\begin{array}{l}\text { Interventional: before and } \\
\text { after survey }\end{array}$ & $\begin{array}{l}\text { After modification of MMC, the } \\
\text { majority of surveyed population } \\
\text { perceives that consensus has } \\
\text { been reached more often }(96 \% \text { of } \\
\text { cases versus } 70 \% \text { cases } p<0.01) \\
\text { especially for avoidability of } \\
\text { complications }(54 \% \text { of cases } \\
\text { versus } 23 \text { of cases, } p<0.05) \text {. }\end{array}$ \\
\hline $\begin{array}{l}\text { Veldenz et al., } \\
2001[46]\end{array}$ & $\begin{array}{l}\text { Academic } \\
\text { surgery }\end{array}$ & $\begin{array}{l}\text { Determining } \\
\text { educational value of } \\
\text { MMC in surgical } \\
\text { residency program }\end{array}$ & $\begin{array}{l}\text { Goal, structure, } \\
\text { process }\end{array}$ & Retrospective & $\begin{array}{l}\text { A weekly peer-reviewed MMC } \\
\text { provides educational value with } \\
\text { ongoing examination of common } \\
\text { problems encountered in the } \\
\text { delivery of surgical care. }\end{array}$ \\
\hline $\begin{array}{l}\text { Hamby et al., } \\
2000[47]\end{array}$ & $\begin{array}{l}\text { Academic } \\
\text { surgery }\end{array}$ & $\begin{array}{l}\text { Determining the } \\
\text { effectiveness of routine } \\
\text { incorporation of local } \\
\text { practice data in MMC }\end{array}$ & $\begin{array}{l}\text { Goal, structure, } \\
\text { process }\end{array}$ & Prospective: chart review & $\begin{array}{l}\text { Incorporating prospective } \\
\text { outcome data into the MMC } \\
\text { provides increased educational } \\
\text { values and opportunities for } \\
\text { quality improvement. }\end{array}$ \\
\hline $\begin{array}{l}\text { Feldman et al., } \\
1997[48]\end{array}$ & $\begin{array}{l}\text { Academic } \\
\text { surgery }\end{array}$ & $\begin{array}{l}\text { Comparing the } \\
\text { incidence of adverse } \\
\text { outcomes recorded in a } \\
\text { prospective general } \\
\text { surgery database with } \\
\text { that of MMC }\end{array}$ & $\begin{array}{l}\text { Structure, } \\
\text { process }\end{array}$ & Prospective: chart review & $\begin{array}{l}\text { Although most severe } \\
\text { complications }(87.5 \%) \text { are } \\
\text { recorded at MMC, a large } \\
\text { proportion of complications } \\
\text { remain unreported. Rigorous } \\
\text { monitoring of outcomes may } \\
\text { contribute further to } \\
\text { improvements in quality of care. }\end{array}$ \\
\hline $\begin{array}{l}\text { Thompson and } \\
\text { Prior, } 1992[49]\end{array}$ & $\begin{array}{l}\text { Academic } \\
\text { surgery }\end{array}$ & $\begin{array}{l}\text { Determining the role } \\
\text { and efficacy of surgical } \\
\text { MMC in a current } \\
\text { quality assurance } \\
\text { program }\end{array}$ & Goal & $\begin{array}{l}\text { Retrospective: chart } \\
\text { review }\end{array}$ & $\begin{array}{l}\text { Although many adverse events } \\
\text { are not identified by MMC, these } \\
\text { conferences remain an important } \\
\text { component of quality assurance } \\
\text { program. }\end{array}$ \\
\hline $\begin{array}{l}\text { Baele et al., } 1991 \\
\text { [50] }\end{array}$ & $\begin{array}{l}\text { Academic } \\
\text { anesthesia }\end{array}$ & $\begin{array}{l}\text { Describing the format of } \\
\text { MMC in detail }\end{array}$ & $\begin{array}{l}\text { Goal, } \\
\text { structure, } \\
\text { process }\end{array}$ & Prospective: chart review & $\begin{array}{l}\text { MMCs offer a good educational } \\
\text { role for residents through } \\
\text { sharing of experiences, using a } \\
\text { "no-blame" attitude. MMCs } \\
\text { improve prevention of } \\
\text { complications. }\end{array}$ \\
\hline
\end{tabular}

(c)

\begin{tabular}{|c|c|c|c|c|c|}
\hline Study & Setting & Stated objective & Category & Type of study & Main results and conclusion \\
\hline $\begin{array}{l}\text { Szekendi et al., } \\
2010[16]\end{array}$ & Academic & $\begin{array}{l}\text { Sharing the authors' } \\
\text { experience with a } \\
\text { patient safety oriented } \\
\text { MMC over } 7 \text { years }\end{array}$ & $\begin{array}{l}\text { Goal, structure, } \\
\text { process }\end{array}$ & $\begin{array}{l}\text { Interventional: before } \\
\text { and after survey }\end{array}$ & $\begin{array}{l}\text { Shift in staff perceptions of } \\
\text { culture: increased voluntary } \\
\text { reporting (by 66\%), improved } \\
\text { patient safety, and amelioration } \\
\text { of quality of care. }\end{array}$ \\
\hline
\end{tabular}

Describing MMC formats across multiple clinical departments; comparing MMC processes with

Aboumatar et al., Academic previously published 2007 [2] analysis models; and exploring how MMCs could be modified to advance medical education and improve patient care

MMCs vary in structure and process and fail to use known analytic framework. Well conducted MMCs provide valuable educational and quality assurance benefits. MMC should elicit input from all caregivers involved, follow a structured approach to identify system defects, and ensure adequate follow-ups on recommendations. 
(c) Continued.

\begin{tabular}{|c|c|c|c|c|c|}
\hline Study & Setting & Stated objective & Category & Type of study & Main results and conclusion \\
\hline $\begin{array}{l}\text { Pierluissi et al., } \\
2003 \text { [4] }\end{array}$ & Academic & $\begin{array}{l}\text { Determining the } \\
\text { frequency at which } \\
\text { MMCs include } \\
\text { adverse events and } \\
\text { errors; determining } \\
\text { whether errors are } \\
\text { discussed and } \\
\text { attributed to a } \\
\text { particular case }\end{array}$ & Structure, process & $\begin{array}{c}\text { Cross-sectional and } \\
\text { prospective }\end{array}$ & $\begin{array}{l}\text { Cultural difference between } \\
\text { internal medicine and surgery } \\
\text { noted. In internal medicine, } \\
\text { fewer cases are presented (1.5 } \\
\text { versus } 2.7 \text { cases, } p=0.001 \text { ) but } \\
\text { more time is spent on case } \\
\text { presentation and discussion }(34.1 \\
\text { minutes versus } 11.7, p=0.001) \text {. } \\
\text { Fewer cases included adverse } \\
\text { events }(37 \% \text { versus } 72 \% \text {, } \\
p<0.001) \text { or errors ( } 18 \% \text { versus } \\
42 \%, p=0.001) \text {. }\end{array}$ \\
\hline
\end{tabular}

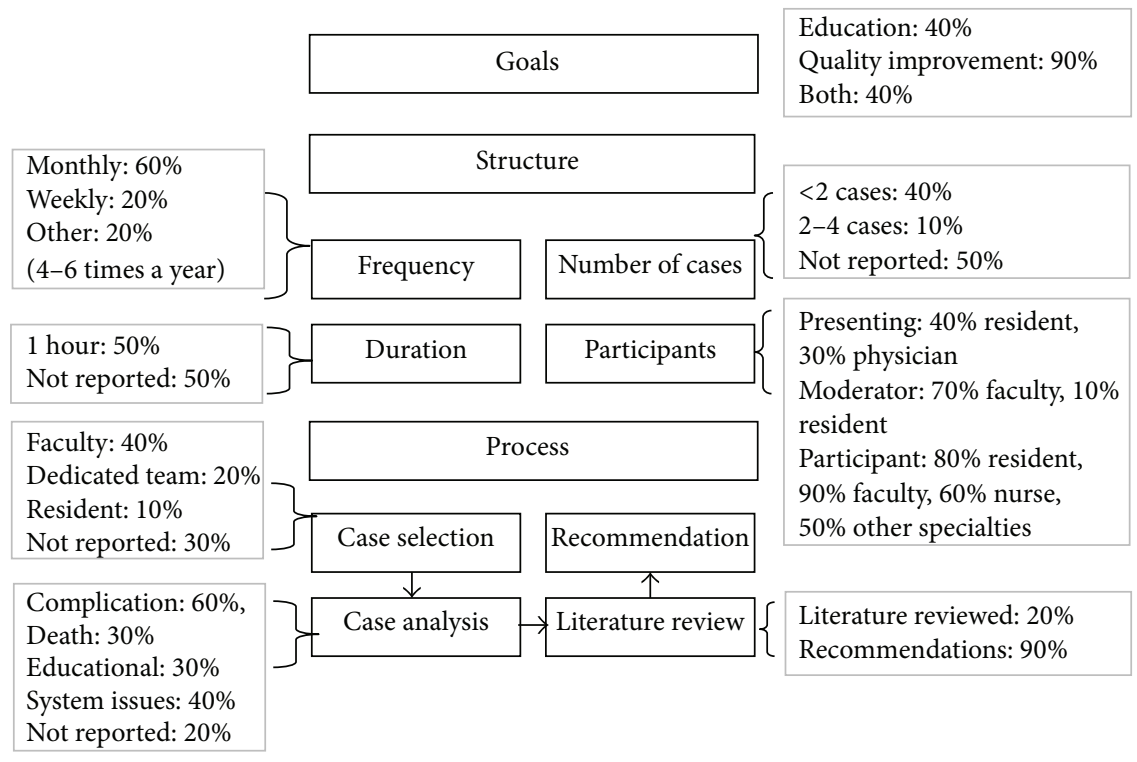

FIGURE 3: Characteristics of MMC in medicine ( $n=10$, percentages not mutually exclusive).

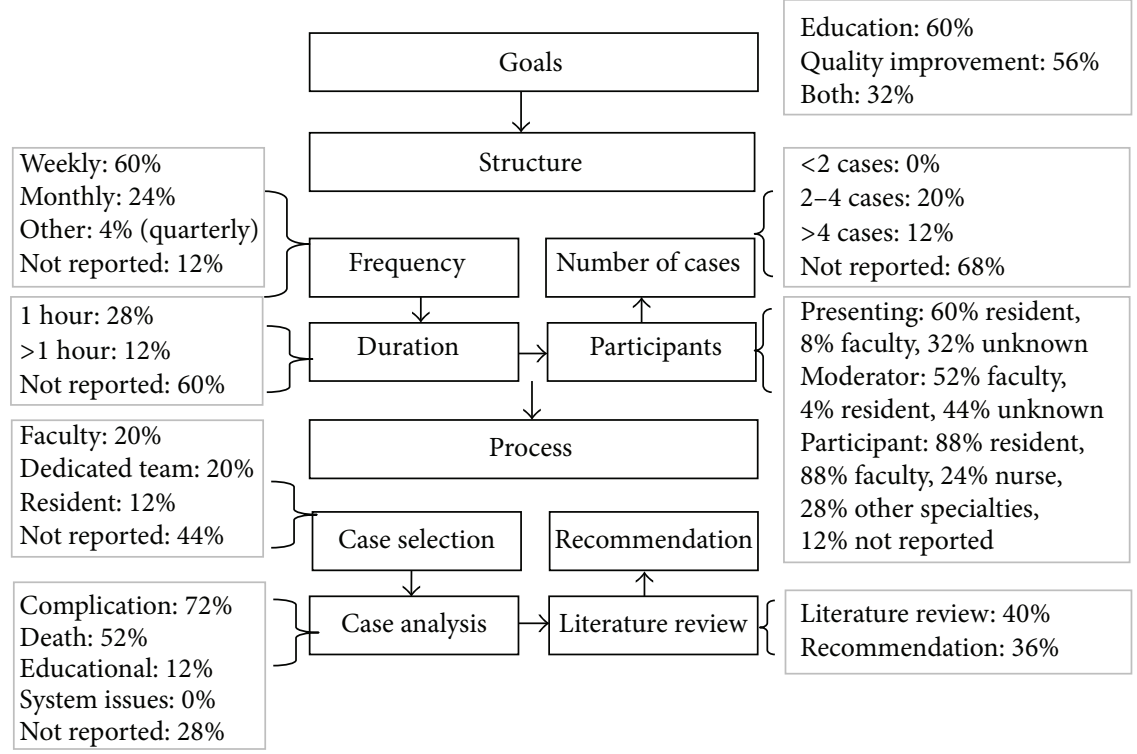

FIGURE 4: Characteristics of MMC in surgery ( $n=25$, percentages not mutually exclusive). 
studies $[2,15,45,53]$ rather suggest to adopt an in-depth analysis of a few selected adverse outcomes. However, even when a majority of adverse outcomes are presented, significant evidence still suggests that MMC underreport complications as compared to other quality assurance databases, such as the National Surgical Quality Improvement Program (NSQIP) $[28,41,42,48]$. Several reasons have been proposed [1, 2, $17,32,54]$ and include a dearth of rigorous definitions of postoperative adverse events, a lack of available resources to facilitate comprehensive data collection, and insufficient time to present all complications.

Although MMCs do not include assessment of all adverse outcomes and errors, their benefit in improving patient care has nevertheless been demonstrated quantitatively in some controlled studies: Antonacci et al. [34, 55] have demonstrated a $40 \%$ decrease in gross mortality over 4 years with rigorous reporting of cases with predefined selection criteria. Similarly, Kirschenbaum et al. [18] have reported a decrease in morbidity and mortality after instituting MMC in the ICU setting. More specifically, significant decreases have been noted in the number of cardiac arrests (3.1/1000 to $0.6 / 1000, p=0.002)$ and all cause deaths $(34 / 1000$ to $24 / 1000, p=0.024)$. These provide quantitative evidence of the quality improvement role fulfilled by MMC $[18,29,32$, 34]. Furthermore, the shift towards providing a safer learning environment with less individual blame $[1,6,14,16,50]$ has encouraged increased staff and resident participation in the MMC process and has led to a more prominent role in medical education $[10,22,31,35,36,39]$. These conclusions, however, are limited by the nonrandomized and qualitative study methodology seen almost uniformly across the studies we examined.

Traditionally, MMCs have consisted of case presentation by a senior resident, followed by staff discussion of itemized problem lists, in order to systematically identify each underlying issue with the goal of preventing future error $[1,53]$. However, it has been demonstrated in the aviation industry that this type of process is not adequate, nor ideal, to capture and respond to error, specifically related to system issues $[14,56]$. Root cause analysis has been proposed as a means to identify system failures and look for potential solutions $[2,56]$. Root cause analysis has been described in detail by Vincent et al. [57, 58]. Essentially, this type of analysis provides physicians with a more structured framework to improve patient safety. Their proposed framework is as follows: identification of the adverse event, why the event occurred (consisting of an analysis of different factors, related to the patient, the task, the caregiver team, the information technology, and the local and institutional environment), implementation of interventions to reduce the probability of its reoccurrence, and finally evaluation of the effectiveness of these interventions. Other methods of analysis have been proposed as well, such as the Association of Litigation and Risk Management (ALARM) method [58, 59]. The ALARM method is limited due to a lack of direct evidence in the literature; the usefulness of this analytical framework in MMC, although promising, has yet to be characterized.

\section{Proposed Guidelines}

Based on the heterogeneous nature of the available literature, although it is difficult to synthesize evidence-based recommendations, some suggestions for the conduct of MMC can certainly be proposed.

5.1. Goals. The goals should be both quality improvement and education. The MMC should be organized such that an optimal balance is maintained at each MMC within a given department. It should also be noted that these goals are not always mutually exclusive and are often complimentary.

5.2. Structure. As there is no strong evidence for the frequency of MMC, the traditional frequency of monthly MMC in medical departments and weekly MMC in surgical specialties may be appropriate. Instead of commenting on a recommended frequency, we propose that each department evaluates whether monthly or weekly conferences are adequate for meeting the services' desired goals, while balancing the other priorities of the department (such as time management, resident education, and patient safety). Similar recommendations can be made with regard to the number of cases discussed or the duration of the MMCs. Arguably, the participants ought to be multidisciplinary, particularly as solutions to systematic problems, usually necessitate a multipronged approach. The presence of a formally recognized facilitator, who moderates the participation of the various members, would enhance MMC outcomes.

5.3. Process. Cases should be selected with predefined criteria or using other existing complication database registries. The cases selected should include both preventable and nonpreventable adverse outcomes, cases with opportunity for quality improvement, cases with educational values, or rare events. For each event presented, we suggest an analysis based on a framework such as the root cause analysis model, to improve effectiveness in identifying both individual and systemic factors. We also suggest appropriate incorporation of evidence-based medicine as well as initiation of quality improvement recommendations during these conferences.

\section{Conclusion}

Patient safety is of vital importance in the practice of medicine. Both medical and surgical services aim to improve patient safety through Morbidity and Mortality Conferences. Although there is a paucity of evidence with regard to their effect on hard outcomes, they are arguably a fundamental tool for achieving important goals in education and quality improvement. Using a unifying conceptual framework for the content and process of MMC, we attempt to summarize the existing literature in a simple, consistent, and reproducible fashion. It is clear that further research is needed to assess the use of different available frameworks to improve the effectiveness of MMC for both medical education and patient safety purposes. 


\section{Competing Interests}

The authors declare that there are no competing interests regarding the publication of this paper.

\section{References}

[1] G. Bal, S. David, E. Sellier, and P. François, "Assessment of morbidity and mortality conferences as a tool for physician education and improvement of quality of care and patient safety: a literature review," La Presse Médicale, vol. 39, no. 2, pp. 161-168, 2010 (French).

[2] H. J. Aboumatar, C. G. Blackledge Jr., C. Dickson, E. Heitmiller, J. Freischlag, and P. J. Pronovost, "A descriptive study of morbidity and mortality conferences and their conformity to medical incident analysis models: results of the morbidity and mortality conference improvement study, phase 1," American Journal of Medical Quality, vol. 22, no. 4, pp. 232-238, 2007.

[3] J. D. Orlander, T. W. Barber, and B. G. Fincke, "The morbidity and mortality conference: the delicate nature of learning from error," Academic Medicine, vol. 77, no. 10, pp. 1001-1006, 2002.

[4] E. Pierluissi, M. A. Fischer, A. R. Campbell, and C. S. Landefeld, "Discussion of medical errors in morbidity and mortality conferences," The Journal of the American Medical Association, vol. 290, no. 21, pp. 2838-2842, 2003.

[5] G. Vidal-Trécan, B. Christoforov, and É. Papiernik, "Mortalitymorbidity conferences: evaluation of an intervention to expand their practice in a university hospital," Presse Medicale, vol. 36, no. 10, part 1, pp. 1378-1384, 2007.

[6] L. T. Kohn, J. M. Corrigan, and M. S. Donaldson, Eds., To Err Is Human: Building a Safer Health System, National Academy Press, Washington, DC, USA, 2000.

[7] "Proceedings of conference on hospital standardization. Joint session of committee on standards," Bulletin of the American College of Surgeons, vol. 3, article 1, 1917.

[8] S. P. Harbison and G. Regehr, "Faculty and resident opinions regarding the role of morbidity and mortality conference," American Journal of Surgery, vol. 177, no. 2, pp. 136-139, 1999.

[9] R. A. Brand, "Ernest Amory Codman, MD, 1869-1940," Clinical Orthopaedics and Related Research, vol. 467, no. 11, pp. 27632765, 2009.

[10] Accreditation Council for Graduate Medical Education, ACGME Program Requirements for Graduate Medical Education in Internal Medicine, Accreditation Council for Graduate Medical Education, Chicago, Ill, USA, 2009.

[11] Accreditation Council for Graduate Medical Education, ACGME Program Requirements for Graduate Medical Education in General Surgery, 2012.

[12] S. M. Berenholtz, T. L. Hartsell, and P. J. Pronovost, "Learning from defects to enhance morbidity and mortality conferences," American Journal of Medical Quality, vol. 24, no. 3, pp. 192-195, 2009.

[13] V. Liu, "Error in medicine: the role of the morbidity and mortality conference," Virtual Mentor, vol. 7, no. 4, 2005.

[14] L. L. Leape, "Error in medicine," The Journal of the American Medical Association, vol. 272, no. 23, pp. 1851-1857, 1994.

[15] C. Kim, M. D. Fetters, and D. W. Gorenflo, "Residency education through the family medicine morbidity and mortality conference," Family Medicine, vol. 38, no. 8, pp. 550-555, 2006.

[16] M. K. Szekendi, C. Barnard, J. Creamer, and G. A. Noskin, "Using patient safety morbidity and mortality conferences to promote transparency and a culture of safety," Joint Commission Journal on Quality and Patient Safety, vol. 36, no. 1, pp. 3-9, 2010.

[17] B. Denis, M. Ben Abdelghani, A. Peter, A.-M. Weiss, J. Bottlaender, and J. Goineau, "Two years of mortality and morbidity conferences in a hospital gastrointestinal endoscopy unit," Gastroenterologie Clinique et Biologique, vol. 27, no. 12, pp. 11001104, 2003.

[18] L. Kirschenbaum, S. Kurtz, and M. Astiz, "Improved clinical outcomes combining house staff self-assessment with an auditbased quality improvement program," Journal of General Internal Medicine, vol. 25, no. 10, pp. 1078-1082, 2010.

[19] H. Ksouri, P.-Y. Balanant, J.-M. Tadié et al., "Impact of morbidity and mortality conferences on analysis of mortality and critical events in intensive care practice," American Journal of Critical Care, vol. 19, no. 2, pp. 135-145, 2010.

[20] A. Kuper, N. Z. Nedden, E. Etchells, S. Shadowitz, and S. Reeves, "Teaching and learning in morbidity and mortality rounds: an ethnographic study," Medical Education, vol. 44, no. 6, pp. 559$569,2010$.

[21] J. H. Szostek, M. L. Wieland, L. L. Loertscher et al., "A systems approach to morbidity and mortality conference," The American Journal of Medicine, vol. 123, no. 7, pp. 663-668, 2010.

[22] M. L. Bechtold, S. Scott, K. C. Dellsperger, L. W. Hall, K. Nelson, and K. R. Cox, "Educational quality improvement report: outcomes from a revised morbidity and mortality format that emphasised patient safety," Postgraduate Medical Journal, vol. 84, no. 990, pp. 211-216, 2008.

[23] A. G. Hasan and W. R. Brown, "A model for mortality-morbidity conferences in gastroenterology," Gastrointestinal Endoscopy, vol. 67, no. 3, pp. 515-518, 2008.

[24] R. C. Goldszer, E. Rittenberg, T. K. Gandhi et al., "Primary care morbidity and mortality conference: new use of an old process," Journal of Clinical Outcomes Management, vol. 13, no. 5, pp. 288291, 2006.

[25] S. J. Kravet, E. Howell, and S. M. Wright, "Morbidity and mortality conference, grand rounds, and the ACGME's core competencies," Journal of General Internal Medicine, vol. 21, no. 11, pp. 1192-1194, 2006.

[26] P. C. Esselman and J. Dillman-Long, "Morbidity and management conference: an approach to quality improvement in brain injury rehabilitation," Journal of Head Trauma Rehabilitation, vol. 17, no. 3, pp. 257-262, 2002.

[27] J. L. Falcone and A. R. Watson, "Surgical Morbidity and Mortality Conference using teleconferencing allows for increased faculty participation and moderation from satellite campuses and saves costs," Journal of Surgical Education, vol. 69, no. 1, pp. 58-62, 2012.

[28] J. L. Falcone, K. K. W. Lee, T. R. Billiar, and G. G. Hamad, "Practice-based learning and improvement: a two-year experience with the reporting of morbidity and mortality cases by general surgery residents," Journal of Surgical Education, vol. 69, no. 3, pp. 385-392, 2012.

[29] M. K. Thomas, R. J. McDonald, E. F. Foley, and S. M. Weber, "Educational value of morbidity and mortality (M\&M) conferences: are minor complications important?" Journal of Surgical Education, vol. 69, no. 3, pp. 326-329, 2012.

[30] K. S. Bevis, J. M. Straughn, J. E. Kendrick, J. Walsh-Covarrubias, and L. C. Kilgore, "Morbidity and mortality conference in obstetrics and gynecology: a tool for addressing the 6 core competencies," Journal of Graduate Medical Education, vol. 3, no. 1, pp. 100-103, 2011. 
[31] R. M. Kauffmann, M. P. Landman, J. Shelton et al., "The use of a multidisciplinary morbidity and mortality conference to incorporate ACGME general competencies," Journal of Surgical Education, vol. 68, no. 4, pp. 303-308, 2011.

[32] M. J. Kim, F. J. Fleming, J. H. Peters, R. M. Salloum, J. R. Monson, and M. E. Eghbali, "Improvement in educational effectiveness of morbidity and mortality conferences with structured presentation and analysis of complications," Journal of Surgical Education, vol. 67, no. 6, pp. 400-405, 2010.

[33] H.-J. Steiger, W. Stummer, and D. Hänggi, "Can systematic analysis of morbidity and mortality reduce complication rates in neurosurgery?" Acta Neurochirurgica, vol. 152, no. 12, pp. 20132019, 2010.

[34] A. C. Antonacci, S. Lam, V. Lavarias, P. Homel, and R. A. Eavey, "A report card system using error profile analysis and concurrent morbidity and mortality review: surgical outcome analysis, part II," Journal of Surgical Research, vol. 153, no. 1, pp. 95-104, 2009.

[35] L. C. Bender, M. E. Klingensmith, B. D. Freeman et al., "Anonymous group peer review in surgery morbidity and mortality conference," The American Journal of Surgery, vol. 198, no. 2, pp. 270-276, 2009.

[36] S. Dissanaike, M. Berry, J. Ginos, R. Paige, W. McNabb, and J. Griswold, "Variations in the perception of trauma-related complications between attending surgeons, surgery residents, critical care nurses, and medical students," American Journal of Surgery, vol. 197, no. 6, pp. 764-768, 2009.

[37] E. Greco, M. Englesakis, A. Faulkner, B. Trojan, L. E. Rotstein, and D. R. Urbach, "Clinical librarian attendance at general surgery quality of care rounds (morbidity and mortality conference)," Surgical Innovation, vol. 16, no. 3, pp. 266-269, 2009.

[38] M. A. Folcik, O. C. Kirton, and M. E. Ivy, "A two-tiered quality management program: morbidity and mortality conference data applied to resident education," Connecticut Medicine, vol. 71, no. 8, pp. 471-478, 2007.

[39] J. M. Prince, R. Vallabhaneni, M. S. Zenati et al., "Increased interactive format for morbidity \& mortality conference improves educational value and enhances confidence," Journal of Surgical Education, vol. 64, no. 5, pp. 266-272, 2007.

[40] M. A. Goldfarb and T. Baker, "An eight-year analysis of surgical morbidity and mortality: data and solutions," The American Surgeon, vol. 72, no. 11, pp. 1070-1081, 2006.

[41] M. M. Hutter, K. S. Rowell, L. A. Devaney et al., "Identification of surgical complications and deaths: an assessment of the traditional surgical morbidity and mortality conference compared with the american college of surgeons-national surgical quality improvement program," Journal of the American College of Surgeons, vol. 203, no. 5, pp. 618-624, 2006.

[42] D. C. Miller, C. P. Filson, L. P. Wallner, J. E. Montie, D. A. Campbell, and J. T. Wei, "Comparing performance of Morbidity and Mortality Conference and National Surgical Quality Improvement Program for detection of complications after urologic surgery," Urology, vol. 68, no. 5, pp. 931-937, 2006.

[43] J. C. Rosenfeld, "Using the morbidity and mortality conference to teach and assess the ACGME General Competencies," Current Surgery, vol. 62, no. 6, pp. 664-669, 2005.

[44] K. M. Murayama, A. M. Derossis, D. A. DaRosa, H. B. Sherman, and J. P. Fryer, "A critical evaluation of the morbidity and mortality conference," American Journal of Surgery, vol. 183, no. 3, pp. 246-250, 2002.
[45] D. A. Risucci, T. Sullivan, S. DiRusso, and J. A. Savino, "Assessing educational validity of the Morbidity and Mortality Conference: a pilot study," Current Surgery, vol. 60, no. 2, pp. 204-209, 2003.

[46] H. C. Veldenz, P. S. Dovgan, M. S. Schinco, and J. J. Tepas III, "Morbidity and mortality conference: enhancing delivery of surgery residency curricula," Current Surgery, vol. 58, no. 6, pp. 580-582, 2001.

[47] L. S. Hamby, J. D. Birkmeyer, C. Birkmeyer, J. A. Alksnitis, L. Ryder, and R. Dow, "Using prospective outcomes data to improve morbidity and mortality conferences," Current Surgery, vol. 57, no. 4, pp. 384-388, 2000.

[48] L. Feldman, J. Barkun, A. Barkun, J. Sampalis, and L. Rosenberg, "Measuring postoperative complications in general surgery patients using an outcomes-based strategy: comparison with complications presented at morbidity and mortality rounds," Surgery, vol. 122, no. 4, pp. 711-720, 1997.

[49] J. S. Thompson and M. A. Prior, "Quality assurance and morbidity and mortality conference," Journal of Surgical Research, vol. 52, no. 2, pp. 97-100, 1992.

[50] P. L. Baele, F. A. Veyckemans, and B. F. Gribomont, "Mortality and morbidity conferences in a teaching anesthesia department," Acta Anaesthesiologica Belgica, vol. 42, no. 3, pp. 133-147, 1991.

[51] J. D. Orlander and B. G. Fincke, "Morbidity and mortality conference: a survey of academic internal medicine departments," Journal of General Internal Medicine, vol. 18, no. 8, pp. 656-658, 2003.

[52] R. A. Gillies, S. Sulkowski, J. Lambert, and P. J. Wagner, "Do morbidity and mortality conferences still have a role in family medicine residencies?" Family Medicine, vol. 39, no. 2, pp. 8283, 2007.

[53] D. C. Gore, "National survey of surgical morbidity and mortality conferences," American Journal of Surgery, vol. 191, no. 5, pp. 708-714, 2006.

[54] A. K. Sachdeva and P. G. Blair, "Educating surgery residents in patient safety," Surgical Clinics of North America, vol. 84, no. 6, pp. 1669-1698, 2004.

[55] A. C. Antonacci, S. Lam, V. Lavarias, P. Homel, and R. D. Eavey, "A morbidity and mortality conference-based classification system for adverse events: surgical outcome analysis: part I," Journal of Surgical Research, vol. 147, no. 2, pp. 172-177, 2008.

[56] S. Johna, T. Tang, and M. Saidy, "Patient safety in surgical residency: root cause analysis and the surgical morbidity and mortality conference-case series from clinical practice," The Permanente Journal, vol. 16, no. 1, pp. 67-69, 2012.

[57] C. Vincent, S. Taylor-Adams, and N. Stanhope, "Framework for analysing risk and safety in clinical medicine," The British Medical Journal, vol. 316, no. 7138, pp. 1154-1157, 1998.

[58] C. Vincent, S. Taylor-Adams, E. J. Chapman et al., "How to investigate and analyse clinical incidents: clinical risk unit and association of litigation and risk management protocol," British Medical Journal, vol. 320, no. 7237, pp. 777-781, 2000.

[59] E. Sellier, S. David-Tchouda, G. Bal, and P. François, "Morbidity and mortality conferences: their place in quality assessments," International Journal of Health Care Quality Assurance, vol. 25, no. 3, pp. 189-196, 2012. 


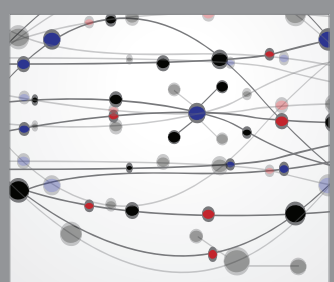

The Scientific World Journal
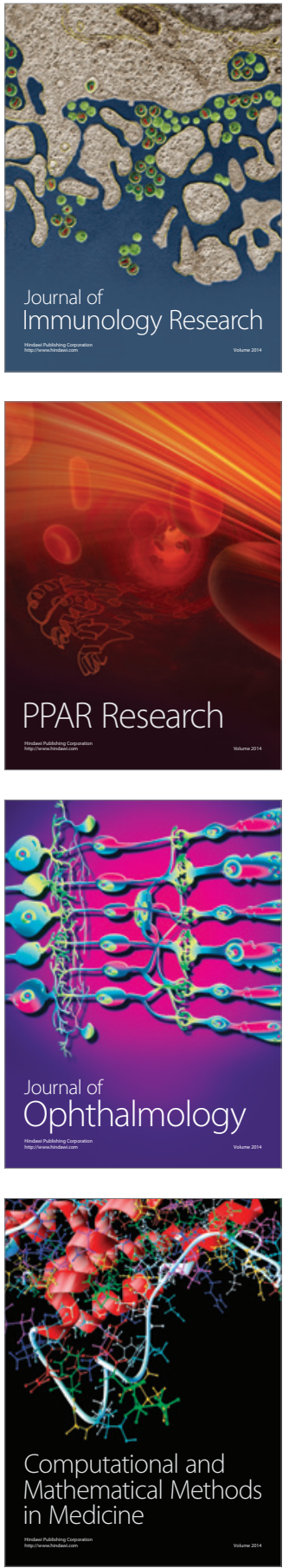

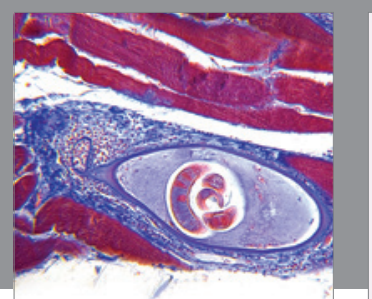

Gastroenterology Research and Practice

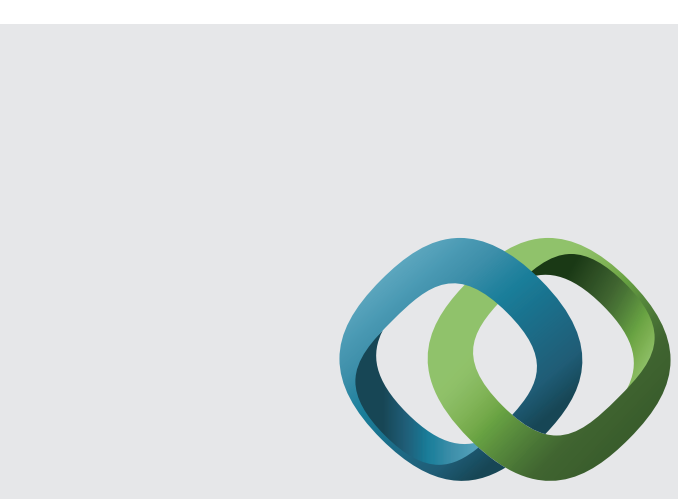

\section{Hindawi}

Submit your manuscripts at

http://www.hindawi.com
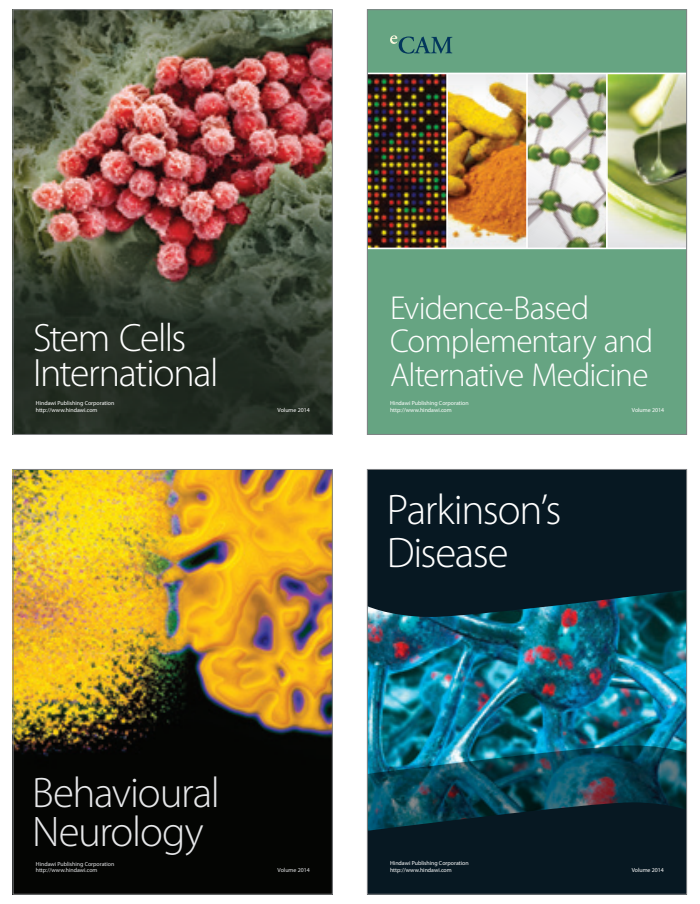
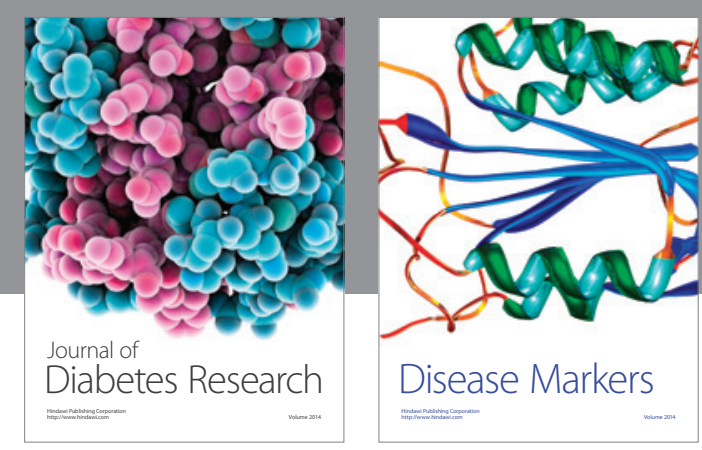

Disease Markers
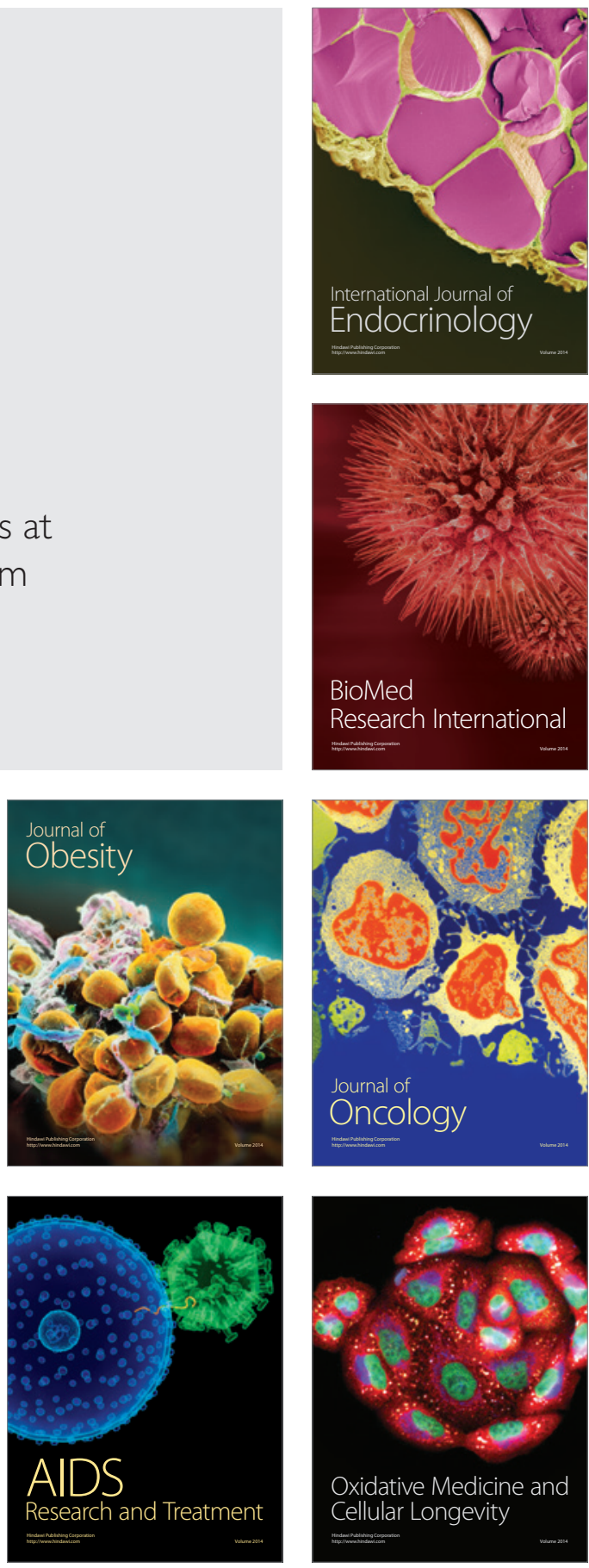